\title{
SlopeX: a plug-in to simplify and fast-track advanced numerical modelling for open pit applications
}

\author{
A Vakili Cavroc Pty Ltd, Australia \\ J Watson Cavroc Pty Ltd, Canada \\ B Abedian Cavroc Pty Ltd, Australia \\ T Styles Cavroc Pty Ltd, UK
}

\begin{abstract}
SlopeX was primarily developed as a plug-in to FLAC3D which helps to reduce the complexity and time taken for beginner to intermediate users to successfully use the software. This plug-in is a collection of several routines, macros and algorithms that automate FLAC3D modelling for open pit mining applications and hence require considerably less expertise to learn and apply.

The software features a sleek and easy-to-navigate user interface with real time help drop-downs. The primary advantage of the plug-in over using unsupported FLAC3D, or any other numerical modelling package, is that model construction time is greatly reduced. The plug-in also uses the built-in constitutive model, improved unified constitutive modelling (IUCM), which is a unique and accurate material model developed specifically for rock response to loading. This model was implemented to be detailed and versatile and has been calibrated and verified against over 100 mining cases worldwide.
\end{abstract}

Keywords: SlopeX, SlopeExamine, improved unified constitutive modelling (IUCM), advanced numerical modelling, slope stability analysis, software, FLAC3D

\section{Introduction}

Numerical modelling is increasingly used in the mining industry to predict the response of the rock mass to various mining activities. With open pits operations and projects going deeper with steeper walls, and facing more complex geological conditions, the traditional state-of-practice analysis, such as limit equilibrium and 2D methods are unable to capture more complex rock mass behaviours.

Nonetheless, state-of-art finite element and finite difference numerical modelling typically requires significant experience and expertise and therefore is rarely applied by site geotechnical practitioners.

As shown by Chiwaye (2010) and many other studies, limit equilibrium (LE) analysis has several limitations when used for open pit design. Compared to LE analysis, explicit numerical modelling software (e.g. FLAC3D) has the following advantages:

- Any failure mode develops naturally; there is no need to specify a range of trial surfaces in advance.

- Multiple failure surfaces (or complex internal yielding) evolve naturally, if the conditions give rise to them.

- Solution consists of mechanisms that are feasible kinematically.

- Finite element or finite difference modelling method is used that accounts for deformations.

- The results of the LE stability analysis can be verified.

Advanced modelling techniques have been used to improve mine performance through optimising various aspects of open pit mines including steepening the pit slopes, targeting slope monitoring and identifying 
areas where opportunities or risks exist or areas where depressurisation are required. However, numerical modelling is often considered 'too difficult' or 'time consuming' and is therefore generally left to specialist consultants with many years of modelling experience. This created the impetus for SlopeExamine (SlopeX).

This paper introduces SlopeX and its key functionalities and how it can be used effectively and routinely as part of the open pit mine design process.

SlopeX was primarily developed as a plug-in to FLAC3D (Itasca 2013) which helps to reduce the complexity and time taken for beginner to intermediate users to successfully use the software. This plug-in is a collection of several routines, macros and algorithms that automate FLAC3D modelling for open pit mining applications and hence require considerably less expertise to learn and apply. Figure 1 shows a conceptual flow chart comparing the conventional FLAC3D numerical modelling process and the SlopeX process.

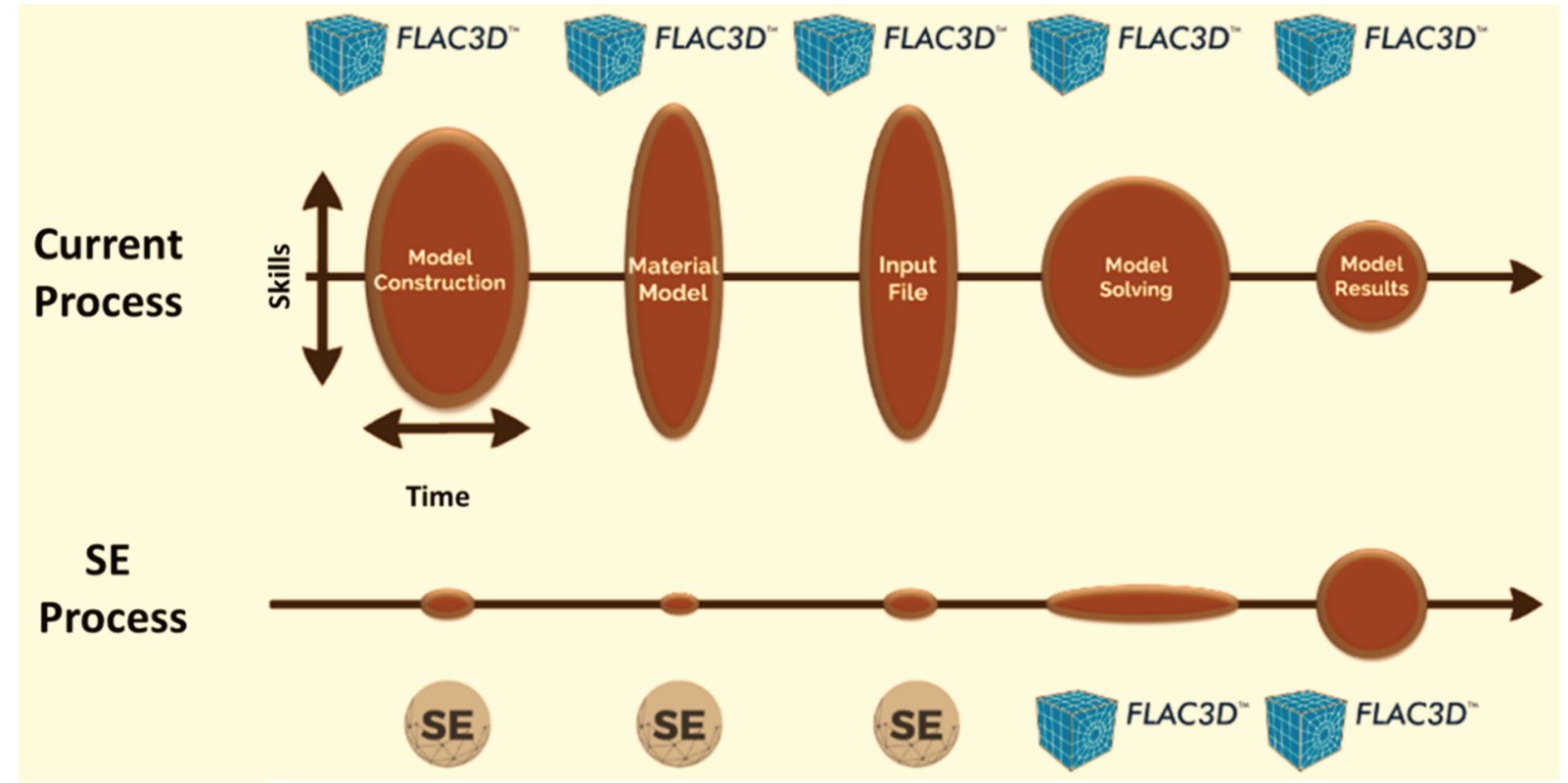

Figure 1 Comparison between conventional FLAC3D modelling process and SlopeX process

The software features a sleek and easy-to-navigate user interface with real time help drop-downs. The primary advantage of the plug-in over using unsupported FLAC3D, or any other numerical modelling package, is that model construction time is greatly reduced. The plug-in also uses the built-in constitutive model, improved unified constitutive modelling (IUCM), a unique and accurate material model developed specifically for rock response to loading. This model was intended to be detailed and versatile and has been calibrated and verified against over 100 mining cases worldwide.

\section{$2 \quad$ Key functionalities}

\subsection{Model setup}

This function allows the user to import several geometrical components into the model including open pit stages, pre-mining topography, area of interest and groundwater table.

Pit stages can be an excavation or the placement of a waste dump. If user select the waste dump option, a surface above the dump material should be imported, while for excavations, a surface below the pit excavation stage should be imported. Dumps can be placed over the surface topography. Groundwater table can be imported separately for each excavation stage. Note that a pre-mining topography can be imported, allowing the analysis of the complete excavation sequence and its interacction with the natural terrain. 
Areas of interest are designed to give the user the additional controls for finer meshing and plotting of a particular area(s) in the pit such as a wall section, underground excavation(s), portal location and surface infrastructure.

Figure 2 shows a simple open pit model which includes pre-mining topography, stage 1 (pit excavation), stage 2 (waste dump) and area of interest (a section of the pit). It should be noted that SlopeX can construct model mesh for more arbitrary shapes having convex and concave areas. In addition, the densification algorithm can identify pit areas with varying floor depth. However, if a different densification is required for pit slopes at certain depths or for specific geological domains, those pit wall sections should be included as areas of interest.
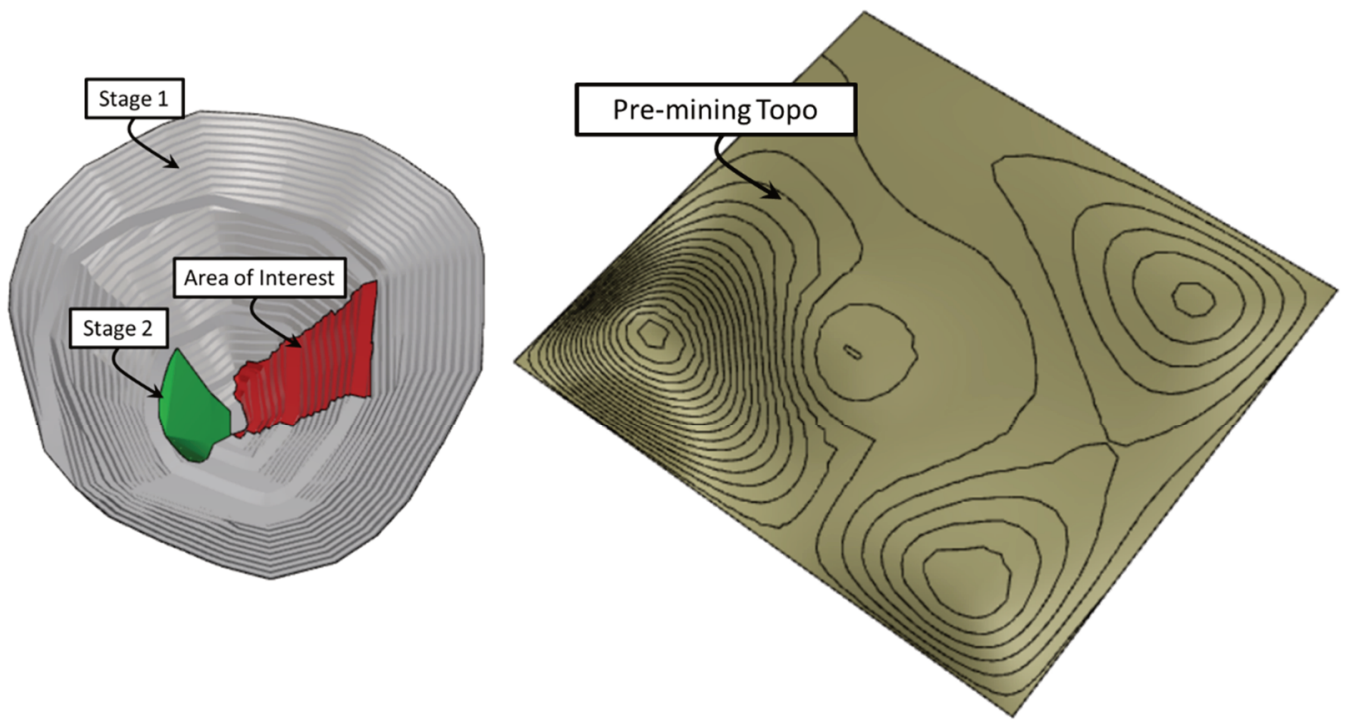

Constructed model using SlopeX in FLAC3D
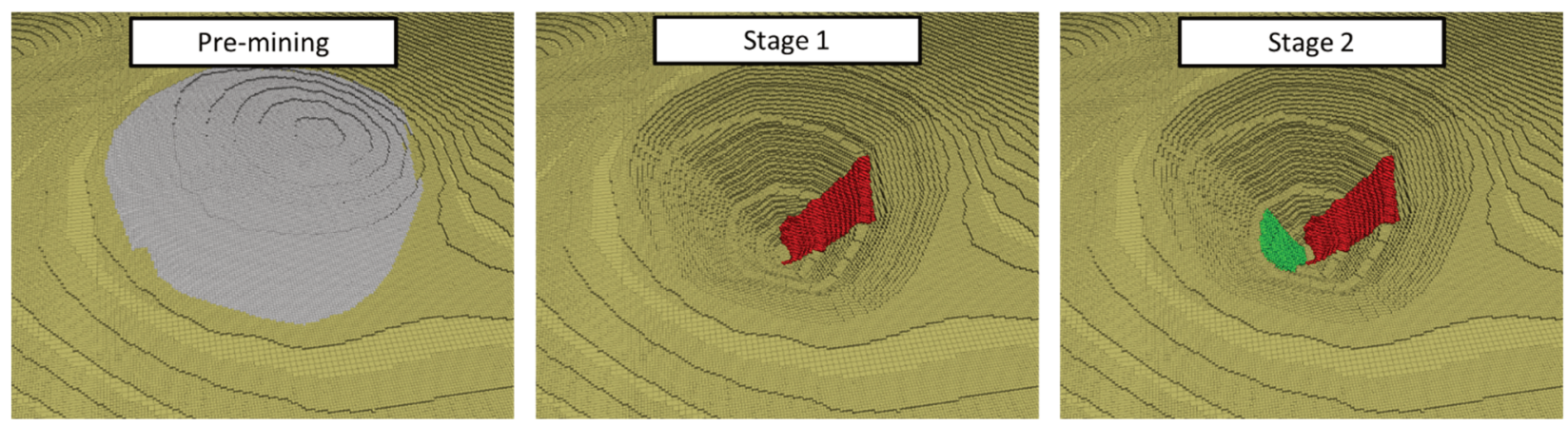

Figure 2 A simple FLAC3D model setup using SlopeX plug-in

\subsection{Automatic meshing}

Meshing is often one of the most time consuming and difficult tasks in finite element and finite difference numerical modelling. Given the importance that mesh plays in model reliability and robustness, it is critical that this aspect be accurate.

SlopeX allows the user to either import a mesh previously constructed in third-party software (e.g. Griddle, Abaqus or Ansys/ICEM) or use SlopeX's built-in routines which streamline the octree meshing process in FLAC3D. An octree mesh is superior to tetrahedral meshing in terms of accuracy of analysis (elements/zones in an octree mesh have aspect ratio of 1 which is ideal). However, the geometrical accuracies are typically not as good as with a tetrahedral mesh, and often requires more time to run and converge.

The automatic octree meshing routine in SlopeX can generate an optimised model mesh using an iterative process, in which the size of the zones increases or decrease in an attempt to find the optimum model mesh. 
The optimum mesh is selected to ensure sufficient resolution for accurate analysis, but also that it does not exceed a user defined maximum computer RAM limit. During the FLAC3D run, if the iteration process was not able to meet user defined inputs (required resolution and computer RAM), a message box will appear and will suggest few alternatives to reiterate the meshing.

For open pit stages, four levels of densification can be assigned: 1 - No Densification, 2 - Minimum, 3 - Densification, 4 - Intermediate Densification.

Level 1 is assigned to pit surfaces which are not of interest but included in the model to capture the correct stress path and softening behaviour. Level 2 assigns a densification that meets the minimum required for a reasonable Factor of Safety calculations. This is based on the recommendations in Read \& Stacey (2009), who found that by experience at least 10 high-order elements are required over the slope height of interest. Levels 3 and 4 densify the zones by 1 and 2 levels respectively. SlopeX routines generate a densified zone automatically that extends over the slope height of interest. The user can also assign a densification distance which controls how far back from the pit crest and pit floor, densification extends to (see Figure 3 ).
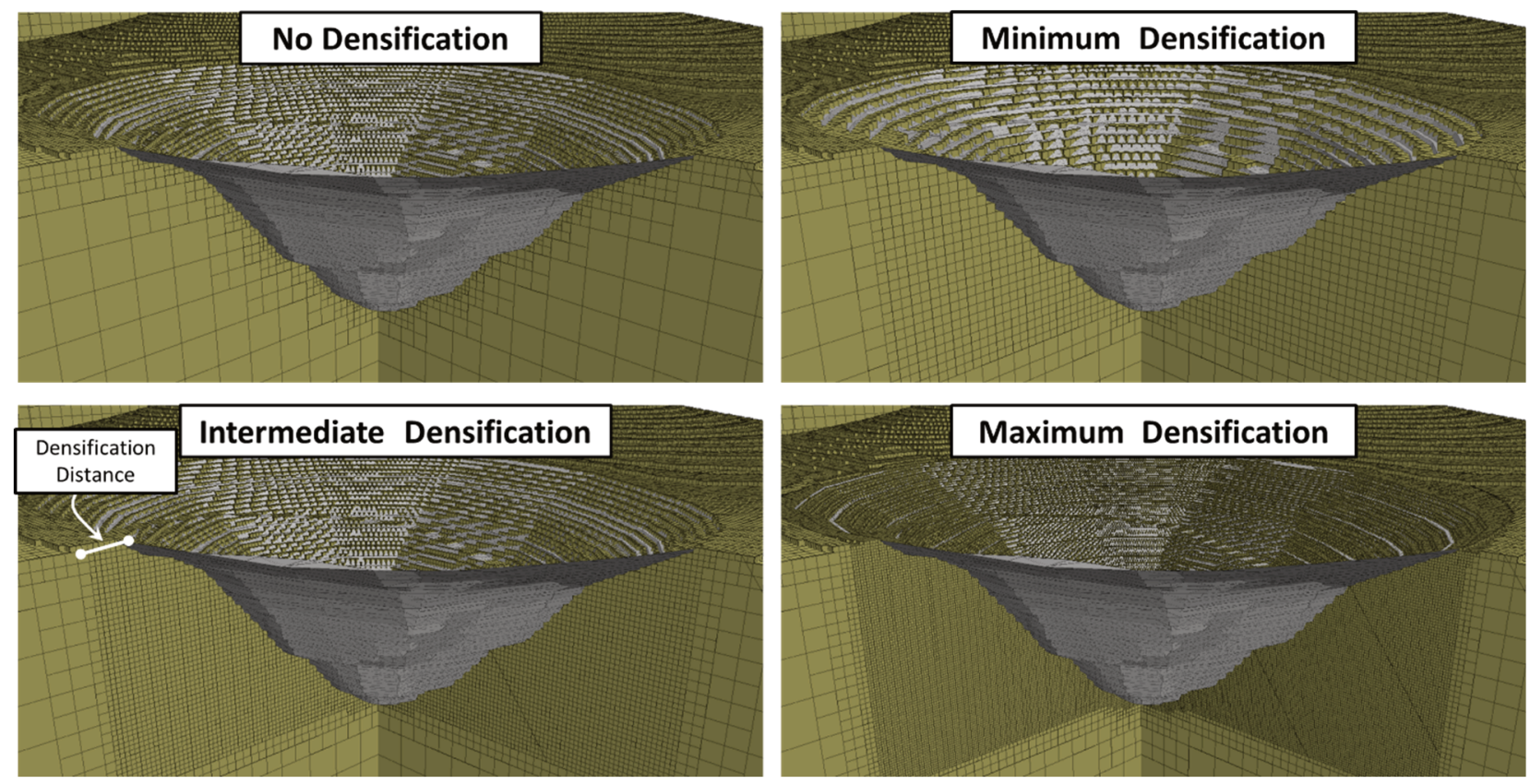

Figure 3 Simple FLAC3D model setup using SlopeX plug-in

For the automatic meshing routines to work effectively, it is important to only include the open pit shell (as excavation stages, if necessary), with any associated topography surfaces removed and include the pre-mining topography surface separately.

\subsection{Improved unified constitutive model}

IUCM (refer to Vakili 2016 for detail) serves as the built-in constitutive model for SlopeX. A constitutive model is a set of rules that dictates how the rock mass within a numerical simulation will behave. The current version of the IUCM has been implemented into Itasca's finite difference code FLAC3D. The model is able to capture particular failure mechanisms and complexities that are typically ignored in commercially available Mohr-Coulomb, bi-linear Mohr-Coulomb or Hoek-Brown constitutive models. The model provides advantages and model complexities as described below.

The IUCM uses a continuous Mohr-Coulomb approximation of the Hoek-Brown failure envelope, instead of the linear or bi-linear approximations that are typically used. Linear approximations of the Hoek-Brown criterion are prone to considerable numerical errors in both high and low stress conditions, depending on how the linearisation is chosen. For open pit applications, the IUCM can account for both the lower stress conditions expected in individual benches or crests, as well as the higher stressed abutments, responsible for 
deep seated failure with a single criterion. The continuous nature of the IUCM provides considerably less conservative simulations compared to linear approximations, and provides a more accurate representation of the rock mass strength throughout the entire open pit. As the failure criterion is continuous, the IUCM also reverts to a linear Mohr-Coulomb envelope for residual state of completely broken and crushed rock.

The model explicitly includes rock strength anisotropy using a nonlinear ubiquitous joint model. This approach accounts for the known directional strength of rock, assigning unique Hoek-Brown failure envelopes to the intact rock, and the foliation or plane of weakness separately. The IUCM's approach to anisotropy contrasts with the conventional approach where the foliation or plane of weakness is defined using a linear Mohr-Coulomb envelope. The nonlinear envelope used in the IUCM can potentially provide a less conservative and more accurate strength estimates in anisotropic rock masses.

The critical plastic strain (i.e. the softening response) in the IUCM is governed by the equations proposed by Lorig \& Varona (2013), derived as part of the International Caving Study. The inclusion of this relationship means that the IUCM corrects for some zone dependencies, particularly those associated with the post-peak softening influences.

The dilation angle in the IUCM is determined using the relationship proposed by Alejano \& Alonso (2005). Their relationship estimates the current peak dilation angle, as a function of the acting confining stress, internal friction angle and the uniaxial compressive strength (UCS). The dependency of dilation on the acting confining stress produces high dilation angles at low confinement, and low dilation angles at high confinement. The dilation angle also softens within the IUCM ultimately reducing to zero. This dynamic behaviour for dilation is also modelled to follow the observations in laboratory rock testing.

The IUCM does not have a fixed post-peak model (softening table). All transient post-peak strength parameters are calculated as a function of the acting confining stress and loading history. The confinement dependency of the rock damage is processed at various stages from peak to residual. Confinement dependency is a major factor controlling rock damage; however, it is largely ignored in all other commercially available constitutive models. For low confining stresses, the IUCM replicates a cohesion and friction strain softening response, while at high confining stresses, the IUCM replicates the observed cohesion softening and friction hardening response. This dynamically defined post-peak response allows for progressive failure to occur near the boundary of the excavation and at the same time, it limits the propagation of damage to unrealistic depths.

Practitioners generally assume a constant modulus of elasticity (Young's modulus) for the rock mass, irrespective of its damage state. In reality, when rock undergoes failure and continuous loading, porosity increases with more voids generated within the rock mass. The more porous a rock mass is, the lower its elastic modulus. Reductions to the rock mass' modulus of elasticity, significantly affects the redistribution of stresses around failed areas, including the induced confining stresses. The impact of modulus softening can be more pronounced in situations where significant rock mass damage or deformation is expected, for example in deep open pit mines. Reyes-Montes et al. (2012) reviewed and collated the previous literature in this area and presented an empirical relationship between modulus drop and the level of porosity in a rock mass. The IUCM uses this relationship to update the elastic modulus values according to changing porosity values.

As a brief summary, the IUCM aims at collating and accounting for all experimentally and operationally documented rock mass behaviours, as a function of the acting stresses and accumulated strain. The levels of accuracy included within the IUCM, are substantially more than what the typical practitioner would assume. This increased complexity is intended to better reflect the actual rock mass failure process, with the intent of increasing the reliability and validity of numerical modelling approaches.

In addition to IUCM, SlopeX users can also assign perfectly plastic Mohr-Coulomb constitutive model for soil like material.

The original version of IUCM was developed through back-analysis of underground case examples. However, Ford et al. (2020) presented few case studies where IUCM model was used to back analyse the documented 
large-scale slope failures in open pit mines. These case studies confirm the suitability of IUCM for open pit applications as well as underground applications.

\subsection{Pre-mining stress setup}

This function assigns the in situ stresses in the model according to principal stress gradients provided by the user, or a more simplistic method where only horizontal to vertical stress ratios and the trend of the major horizontal stress are provided. The interface allows the user to also include a locked stress for each principal stress component which will add a constant stress value at ground level elevation. This algorithm assigns the stresses differently if the pre-mining topography is included in the model. In this case, stresses will be calculated by using the stress gradients and distance from each face in the topography. This allows a uniform distribution of stresses that follows the different elevations of the topography in mountainous areas (as shown in Figure 4) and can significantly reduce the convergence time. If no topography is included by the user, stresses will be calculated from the zones associated with the pit excavation geometries, which have the greatest elevation.

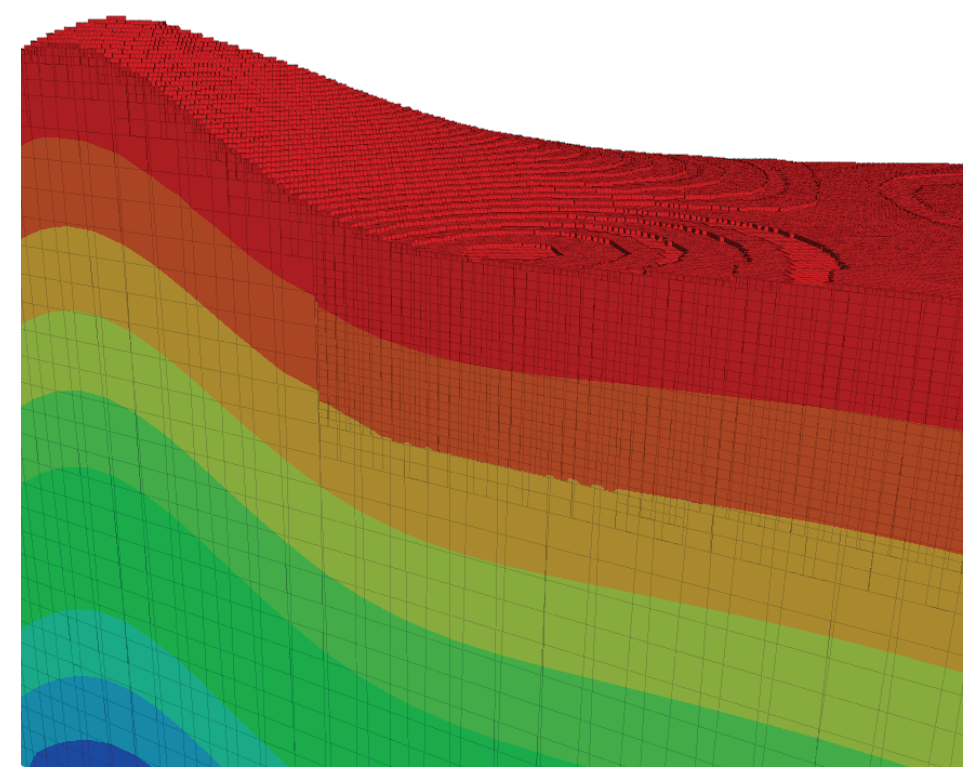

Figure 4 Pre-mining stress initiation in mountainous topography using SlopeX built-in routines

\subsection{Boundary conditions}

This function automatically assigns the appropriate boundary conditions in the model without any major user action. The code first extends the outer boundaries of the model by an offset value provided by the user. The offset value is added to the maximum and minimum extent of the geometries in east, west, north, south, up and down directions which is calculated automatically in this function. No fixed condition is applied to the topography (top) boundary but in case of no topography, the top boundary will be defined by deleting the zones above the upper most elevation of the open pits. The east, west, north and south boundaries are free to move in vertical direction (up or down), while the bottom boundary is fixed in all directions.

\subsection{Pit excavation method}

SlopeX allows the user to excavate each open pit stage either gradually or immediately. The immediate excavation is more suitable for cases where detailed quarterly or yearly pit stages are available and included in the model. However, when pit stages represent longer excavation period or the ultimate pit surface, the gradual excavation should be used to ensure that correct stress path and softening behaviours are captured in the models.

Read \& Stacey (2009) explain the role of load path (or stress path) on slope stability analysis and highlight that for many slopes, stability seems to depend mostly on slope conditions, such as geometry and pore 
pressure distribution at the time of analysis, and very little on the load path taken to get there. They also provide an approach in which only one, two or three excavation stages are modelled. Read \& Stacey (2009) show/suggest that two calculation steps are taken for each stage. In the first step, the model is run elastically to remove any inertial effects caused by sudden removal of a large amount of material. In the second step, the model is run allowing plastic behaviour to develop. They reported that reasonable solutions to many slope stability problems have been obtained with this approach.

When pit excavation stages are too large, the elastic calculation stage may involve stresses well outside the failure envelope for structures and/or rock masses. The return path to admissible stresses may not be realistic in all cases (Read \& Stacey 2009). Therefore, in these cases, the excavation should be done gradually by taking out horizontal slices.

In SlopeX, the solving process involves the calculation steps described above (first elastic and then inelastic) and each step is solved to a standard solve ratio (1.5e-5 for elastic step and 6.5e-6 for inelastic step). The user can define additional cycling if convergence is not fully achieved after the standard solving process.

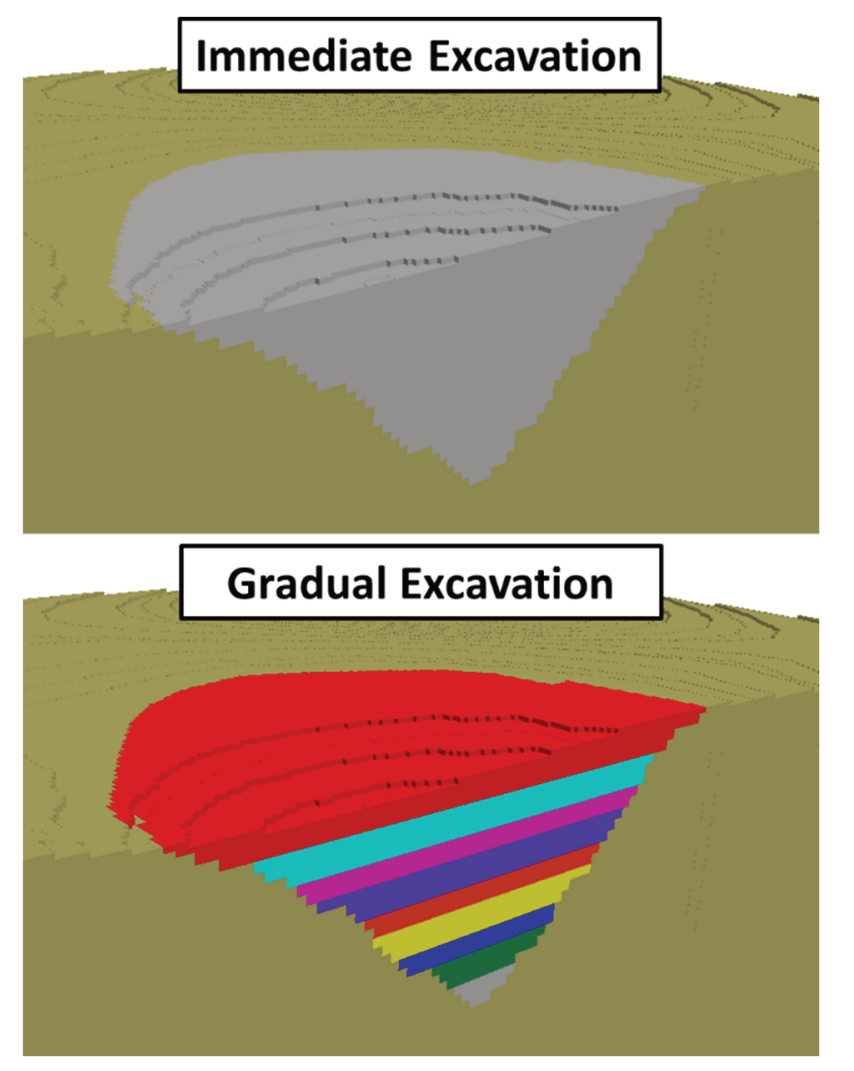

Figure 5 Two different pit excavation method using SlopeX

\subsection{Shear strength reduction for Factor of Safety calculation}

Because IUCM uses a continuous Mohr-Coulomb approximation of the Hoek-Brown failure envelop, the shear strength reduction (SSR) mechanism can follow the conventional method for Factor of Safety (FoS) calculations in which the cohesive strength and friction angle are downgraded. The following modifications are made during each SSR trial:

- Cohesion (c), friction angle $(\phi)$ and tensile strength $(t)$ are downgraded for each zone in the model regardless of the state that they are (peak or residual), using these questions respectively:

$$
c_{\text {trial }}=\left(\frac{1}{\text { FoS }}\right) c ; \varphi_{\text {trial }}=\tan ^{-1}\left(\frac{1}{F o S}\right) \tan \varphi ; t_{\text {trial }}=\left(\frac{1}{F o S}\right) t
$$

- If anisotropic material exists, the above equations are used to downgrade the peak and residual ubiquitous joint properties (c, $\phi$ and $\mathrm{t}$ ). 
- If explicit faults are included in the model (2D interfaces), the interface properties are also downgraded following the same equations.

After each SSR trial, a DXF file of the failed volumes is exported. The failure criterion can be controlled by the user and is based on a displacement and velocity criteria. These DXF files can then be used to visualise the FoS of different parts of the pit in 3D as shown in Figure 6.

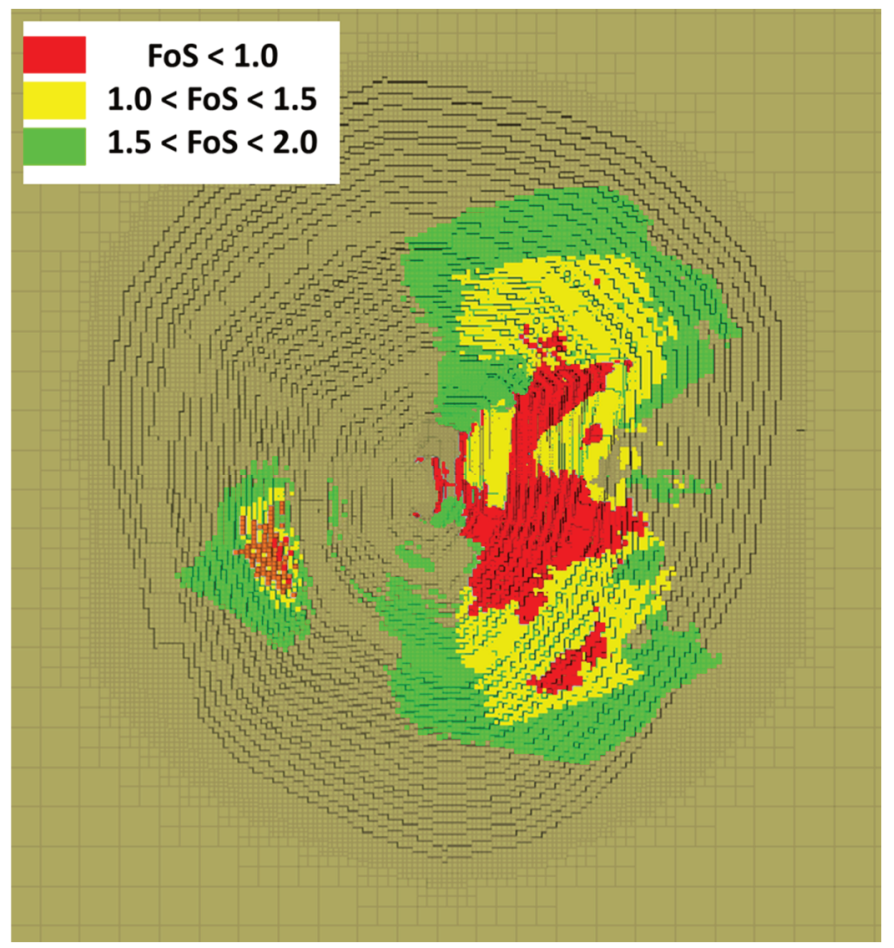

Figure 6 3D visualisation of FoS using the automatic SlopeX outputs

\subsection{Accurate strength anisotropy representation}

Although strength anisotropy is replicated accurately in the IUCM, certain geological formations may require accurate representation of the foliation/bedding/schistosity's orientation. In many cases, folding or faulting can cause significant variation in structural orientations which need to be accounted for in a numerical model to ensure an accurate rock mass behaviour.

SlopeX has a built-in routine that allows the user to import geological wireframes that represent the orientation of foliation, bedding or schistosity so that ubiquitous joint orientations are assigned accordingly in the model. Figure 7 shows an example where this functionality is applied to assign the ubiquitous joint orientations according to the geological surfaces. 


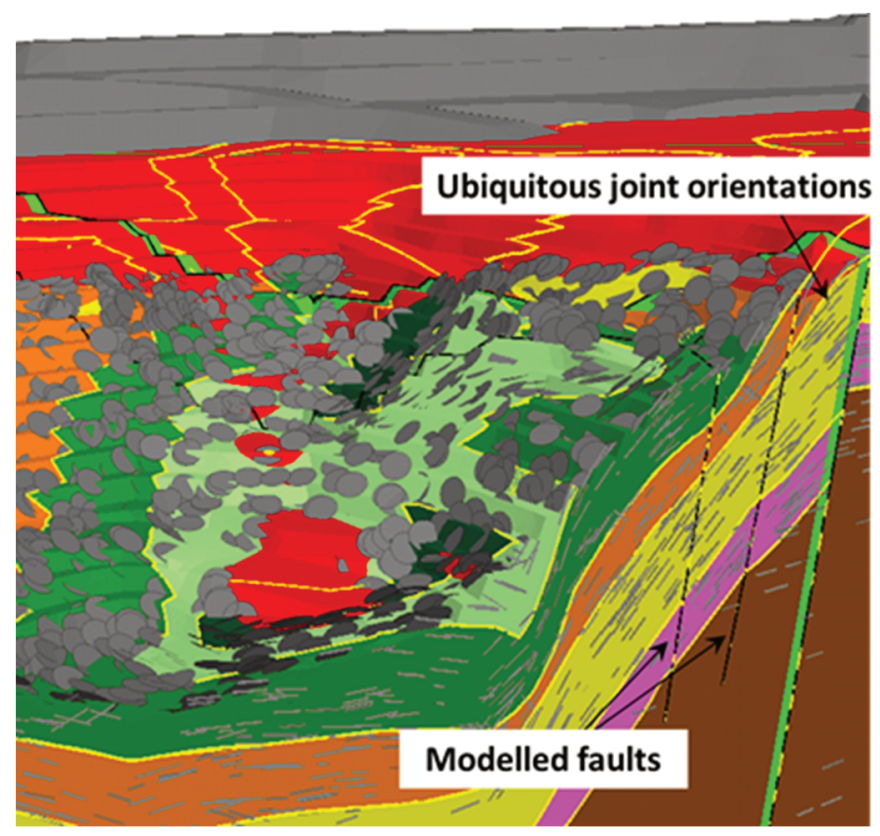

Figure 7 Accurate representation of ubiquitous joints according to geological surfaces

\subsection{Rock mass assignments}

The geotechnical domains and associated properties are assigned through this functionality. Users can import the geometries that define each geotechnical domain (closed volumes or surfaces defining the base of the geological units) and then assign the properties associated with each domain. The compulsory properties required for the model are all readily available from common laboratory tests and rock mass characterisation (e.g. UCS, Ei, GSI etc.). Figure 8 shows an example of an open pit model with complex geological formations constructed using SlopeX.

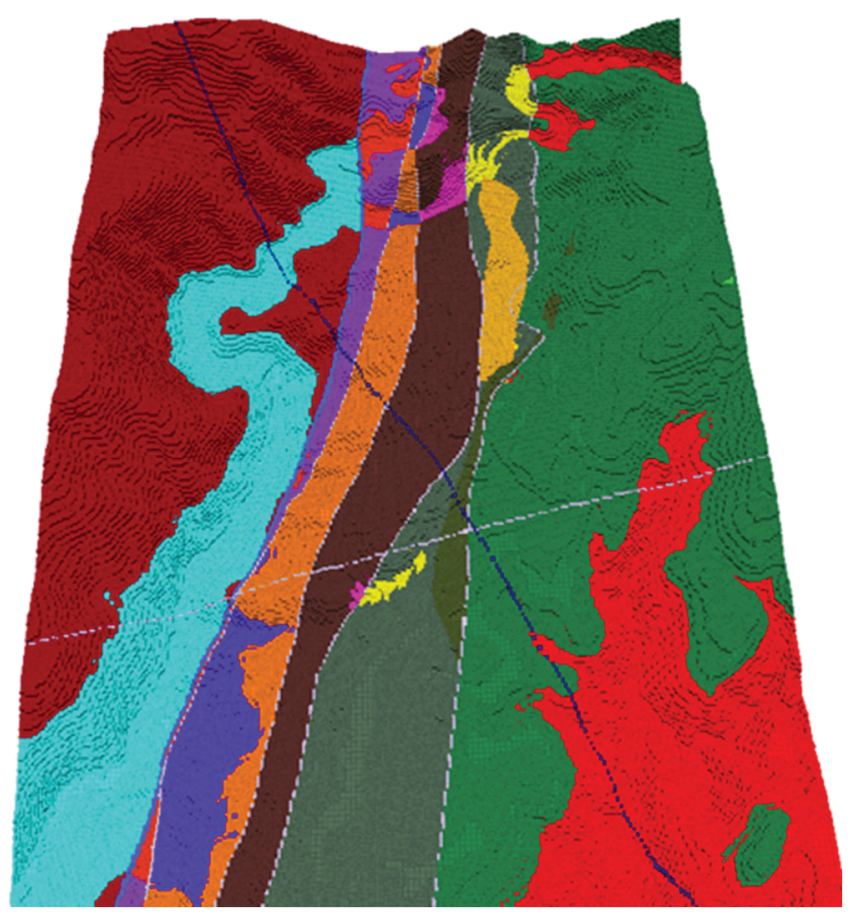

Figure 8 Assignment of geotechnical domains 


\subsection{Fault and structure inclusions}

Faults and other large-scale structures can be included explicitly in models constructed using SlopeX. The user can include up to 20 faults in the model.

For models constructed using the automatic octree meshing routine, the faults will be included as a domain with a user defined thickness. To ensure accurate representation and degree of freedom for the fault to behave realistically, the zones within the given thickness will be densified so that at least three zones exist across the thickness of the fault. The properties of the faults can be assigned either using simple Mohr-Coulomb properties or Hoek-Brown (through IUCM), if it can be justified.

For models constructed using imported mesh from third-party program with structure included explicitly as 2D faces within the mesh, the user can include faults as 2D surfaces that are replicated using Interface elements in FLAC3D. Currently the user can only assign the properties for this type of models using perfectly plastic Mohr-Coulomb parameters.

Figure 9 shows two examples of faults included using SlopeX for octree mesh models and imported tetrahedral mesh.

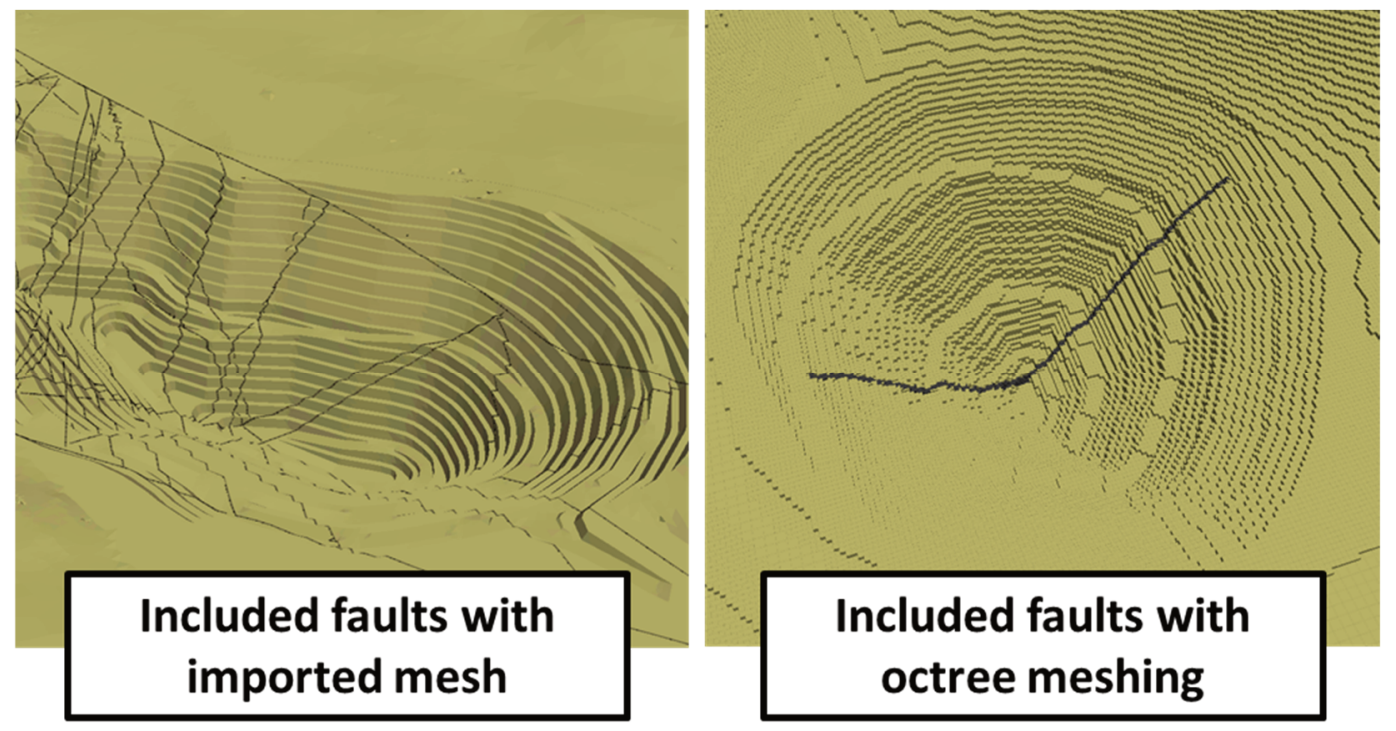

Figure 9 Included faults in an octree mesh and imported mesh

\section{User interface}

SlopeX's user interface (UI) is a desktop app that guides the user to construct a FLAC3D model through the SlopeX platform. The app assists the user to assign the model parameters, upload required geometry files, and control different settings. Input validations and parameter dependencies are built in the UI, which can assist the users to avoid falling into the common pitfalls of setting a model up.

The user journey is designed according to the industry's state-of-art numerical modelling practices which brings about more than 15 years' experience and more than 100 numerical modelling studies for mines around the world. The $\mathrm{UI}$ design process went through multiple design iterations to address shortfalls that were reported by the users during the usability test process. Figure 10 shows three snapshots of SlopeX's UI.

The UI app is built with the latest web technologies (React and Node.js), which can be run as standalone desktop apps on major operating systems but also facilitate easy transition to cloud-based model processing in the future. This approach was chosen to ensure that the app can be installed on a local desktop for users in remote areas with limited or no internet access. 


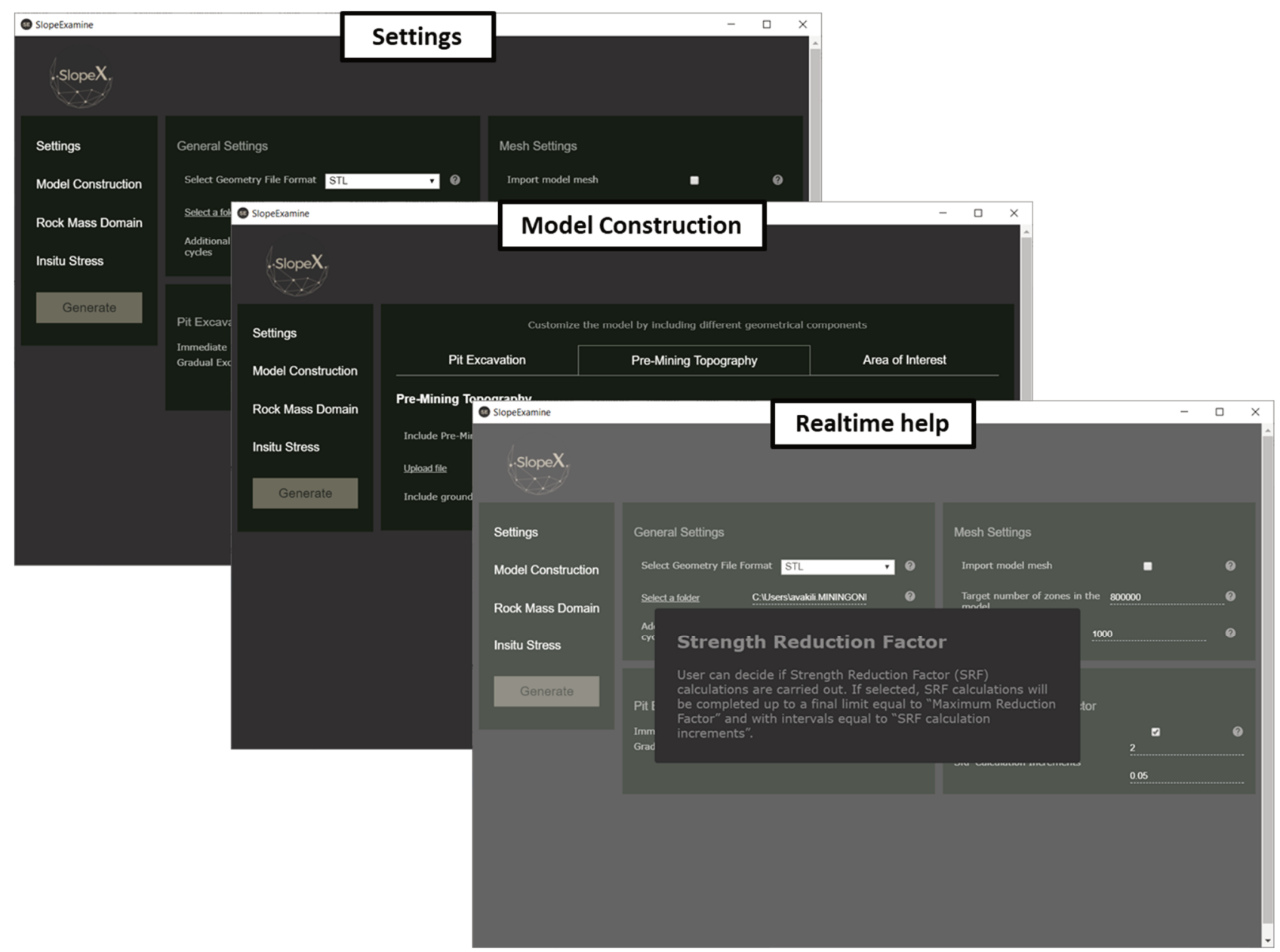

Figure 10 SlopeX's graphical user interface

\section{$4 \quad$ Limitations and assumptions}

The following limitations, assumptions and recommendations should be considered when using SlopeX.

No numerical modelling tool or program can simulate the behaviour of the rock mass $100 \%$ accurately and users should always understand that models are just a representation of reality and are meant to help us understanding more complex physical behaviours. Numerical modelling should never substitute observations and sound engineering judgment and should be used as a tool only.

Detailed limitations and assumption associated with the IUCM are provided in Vakili (2016) and are not repeated here. These limitations should be taken into account when using the SlopeX software.

When using automatic octree meshing logics, it is important to note that long and narrow geometries (e.g. resource or geological domains) may not be represented accurately in the model and refinement of the mesh may be necessary to ensure that they are included in the model accurately.

SlopeX significantly reduce the skills and time required to do advanced numerical modelling and therefore there is a higher likelihood for inexperienced or unqualified engineers to carry out the analysis. Therefore, it is very important for mine sites and consultants to ensure that the users of the software are fully aware of the importance of quality and quantity of data and how to derive the appropriate input parameters. In addition, post-processing and interpretation of the model outputs require experience and good knowledge of geotechnical and mining engineering. However, this applies to any other simple tools like LE or empirical charts for which lack of experience and understanding can lead to unreliable design. 


\section{Conclusion}

This paper described the key functionalities and processes developed for SlopeX, which is a plug-in to FLAC3D. SlopeX was developed with the intention to narrow the gap between state-of-art and state-of-practice numerical modelling practices for open pit applications.

The empirical and the outdated analytical tools are way too simplistic to enable effective prediction of rock mass behaviour in an open pit mine. These conventional methods, at best, are suitable for providing guidelines for slope design in soil and shallow open pits in rock and they have several shortcomings when used in more complex ground conditions or deep open pits.

The authors believe that application of SlopeX in other open pit mines can bring about significant economic and safety benefits. However, this requires a fundamental shift in management culture and avoiding over-reliance on outdated design methods such as benchmarking, empirical or over simplified analysis methods.

Mines are developing into deeper and more challenging geotechnical environments and profit margins are getting narrower. It is impossible to expect that benchmarking and empirical methods or limit equilibrium analysis, which were originally developed for considerably different geotechnical conditions, will be able to guide us for future mine design. Compared to conventional unsupported FLAC3D modelling, SlopeX provides the following benefits:

- SlopeX's built-in constitutive model (IUCM) provides a much more reliable predictions of rock damage than commercially available models in FLAC3D. In more challenging ground conditions and in deeper and larger open pits where brittle intact rock failure is expected, IUCM is the only commercially available model that can simulate the rock mass response accurately.

- Meshing and setting up models in SlopeX are up to 50 times faster and easier than unsupported FLAC3D, which enables more geotechnical engineers including site personnel to carry out advanced numerical modelling more routinely.

\section{Acknowledgement}

SlopeX development would have been impossible without the support of individuals who invested their own time and funds for this project. In particular, we acknowledge Julian Watson and Petram Mechanica LLC who generously sponsored this project.

\section{References}

Alejano, LR \& Alonso, E 2005, 'Considerations of the dilatancy angle in rocks and rock masses', International Journal of Rock Mechanics and Mining Sciences, vol. 42, no. 4, pp. 481-507.

Chiwaye, HT 2010, A Comparison of the Limit Equilibrium and Numerical Modelling Approaches to Risk Analysis for Open Pit Mine Slopes, Doctoral dissertation, University of the Witwatersrand.

Ford, A, Lucas, D \& Vakili, A 2020, 'Validation of the Improved Unified Constitutive Model (IUCM) for open pit applications', in PM Dight (ed.), Proceedings of the 2020 International Symposium on Slope Stability in Open Pit Mining and Civil Engineering, Australian Centre for Geomechanics, Perth, pp. 953-968.

Itasca 2013, FLAC3D, version 5.01, Itasca Consulting Group, Inc., Minneapolis.

Lorig, LJ \& Varona, P 2013, 'Guidelines for numerical modelling of rock support for mines', in Y Potvin \& B Brady (eds), Proceedings of the Seventh International Symposium on Ground Support in Mining and Underground Construction, Australian Centre for Geomechanics, Perth, pp. 81-105.

Read, J \& Stacey, P 2009, Guidelines for Open Pit Slope Design, CSIRO Publishing, Collingwood.

Reyes-Montes, JM, Sainsbury, BA, Andrews, JR \& Young, RP 2012, 'Application of cave-scale rock degradation models in the imaging of the seismogenic zone', Proceedings Sixth International, Conference and Exhibition on Mass Mining, Massmin2012, The Australasian Institute of Mining and Metallurgy, Carlton pp. 10-14.

Vakili, A 2016, 'An improved unified constitutive model for rock material and guidelines for its application in numerical modelling', Computers and Geotechnics, vol. 80, pp. 261-282. 